\title{
Representando el referéndum del Brexit en la prensa británica. Análisis de Encuadre de titulares de cuatro diarios
}

\author{
Representing the Brexit referendum in the British press. \\ framing Analysis of headlines of four newspapers
}

\author{
Lenin Miranda Maldonado \\ Facultad de Ciencias Sociales y Humanas, Universidad Central del Ecuador (Ecuador) \\ Imm_ecuador@hotmail.com, https://orcid.org/0000-0001-5543-1092
}

Recibido: 06-10-2021 Revisado: 02-12-2021 Aceptado: 05-12-2021

\section{Resumen}

La salida del Reino Unido (RU) del bloque de la Unión Europea (UE) ha sido uno de los eventos políticos más turbulentos de esa región en los últimos años. El resultado del referéndum, en el 2016, supuso una fractura importante en uno de los proyectos de integración más exitosos que se han dado a nivel mundial; además, sirvió como campo de batalla, en términos discursivos, donde chocaron diversas visiones respecto a la permanencia del RU en el bloque europeo. Los medios de comunicación participaron activamente en la contienda política a través de representaciones parcializadas de los eventos, disfrazadas de noticias objetivas, que incidieron, de un modo u otro, en la construcción de opinión pública. Este trabajo estudió, a través del Framing Analysis, cómo cuatro medios británicos (Daily Mail, Mail on Sunday, Daily Mirror y Sunday Mirror) encuadraron el debate sobre el Brexit para posicionar una mirada específica del problema. Se pudieron identificar tres grandes estrategias, a favor y en contra de la permanencia, en un total de 60 titulares entre los cuatro periódicos estudiados: el encuadre de la crisis económica, el del intruso y el del proyecto "pánico".

Palabras claves: Brexit, Reino Unido, framing analysis, análisis de discurso, Unión Europea.

\section{Abstract}

The withdrawal of the United Kingdom (UK) from the European Union (EU) bloc has been one of the most turbulent political events that the region has experienced in recent years. The referendum's outcome in 2016 marked an essential fracture in one of the most successful integration projects that has occurred worldwide; furthermore, it served as a battlefield, in discursive terms, where different views collided regarding the permanence of the UK in the European bloc. The media actively participated in the political contest through biased representations of events (disguised as objective news) that would influence the construction of public opinion in one way or another. Through Framing Analysis, this work studies how four British newspapers (Daily Mail, Mail on Sunday, Daily Mirror and Sunday Mirror) framed the debate on Brexit to consolidate a specific view of the problem. It was possible to identify three significant strategies in a total of 60 headlines among the four papers under study: the framing of the economic crisis, the intruder, and the "panic" project.

Keywords: Brexit, United Kingdom, framing analysis, speech analysis, European Union. 


\section{Introducción}

En junio de 2016, ciudadanos británicos votaron por salir de la Unión Europea (UE). Este evento conmocionó a muchos observadores de todo el mundo y abrió un período de incertidumbre política en el Reino Unido (RU), que obligó a David Cameron y a Theresa May a dejar Downing Street. ${ }^{1}$ La ruptura de una de las principales economías de la región, con Europa, significó un golpe a un proyecto de integración que se venía consolidando desde los años 50. Como se ha explorado recientemente en un importante cuerpo de literatura, los medios británicos jugaron un papel fundamental en el resultado del referéndum (Charteris-Black, 2019; Levy et al., 2016; Taylor, 2017). Estas instituciones construyeron relatos e interpretaciones parcializadas respecto a la relación entre el RU y Europa que indujeron a la población a leer este fenómeno desde un punto de vista específico. En ese sentido, es posible señalar que participaron activamente en la confrontación política a partir de sus representaciones sociales. Según Payne (2018), el referéndum del 2016 acentuó una vieja discusión en la sociedad británica que claramente se reflejó en la cobertura mediática, lo que puede leerse en varios estudios (Hinde, 2017; Jackson et al., 2016; Khabaz, 2018; Levy et al., 2016; Maccaferri, 2019; Marsh, 2018; Seaton, 2016; Zappettini yKrzyżanowski, 2019).

Los diarios en el RU, más que en otros países europeos, han participado activamente de la vida política y, durante la campaña del referéndum, declararon abiertamente su apoyo a una de las dos facciones en disputa (Baker et al., 2013; Coxall et al., 2003; Hinde, 2017). Como bien señala Hinde (2017), “durante las últimas décadas, la opinión [en los medios británicos] se ha filtrado cada vez más en los reportes noticiosos, de modo que sobre algunos temas muchos periódicos hacen poca o ninguna pretensión de objetividad” (p. 81). Sin embargo, y a pesar de esta particularidad de la prensa escrita británica, se asume que los medios deben ser una fuente confiable de información, que reporte hechos y no solo opiniones. Si un periódico quiere permanecer en el negocio, y mantener su audiencia, debe construirse en relación con las demandas del lector; es decir, los periodistas tienen que actuar como periodistas, o aparentar serlo, y no mostrarse como la propaganda oficial de ningún partido político. En cierto modo, la legitimidad de un periódico es su principal capital. $^{2}$

Por tanto, y asumiendo que la credibilidad de un medio está en juego, se considera que el proceso de manipulación debe contener formas creativas al elaborar representaciones subjetivas de la realidad. Es decir, este proceso no funciona de manera transparente y obvia, como señalaban los denominados modelos de "influencia directa", actualmente ya desmontados, según los cuales a las personas se les dice qué y cómo pensar respecto a un fenómeno específico (Hall, 2005b). Tomando distancia de esta perspectiva reduccionista, se sostiene que el proceso comunicativo es mucho más complejo: los periódicos deben compartir con sus lectores una interpretación de los hechos sociales que se muestra como la más valiosa, o la única exacta, incluso si se sabe que esta lectura es abiertamente polémica. Para hacerlo, los medios tienden a "enfatizar" u "oscurecer" ciertos aspectos de un evento, para que las audiencias procesen un texto de una manera particular, haciéndolo más notorio, memorable y significativo para el público. A este proceso se le ha denominado encuadre o framing (D’Angelo y Kuypers, 2010; Entman, 1993; Kovecses, 2010; Lakoff, 2003; Reese et al., 2008; Van Gorp, 2005, 2010). En palabras de Entman (1993), "encuadrar es seleccionar algunos aspectos de una realidad y hacerlos más destacados en un texto comunicativo, de tal manera que se promueva una definición particular del problema, una interpretación causal, evaluación moral y/o recomendación de soluciones" (p. 52). Por lo tanto, el encuadre se logra a través de elecciones discursivas, conscientes o inconscientes, materializadas en dispositivos co-

Es una calle de Londres donde reside el primer ministro del Reino Unido.

Al respecto, Baker et al. (2013) comentan: "la cobertura negativa e inexacta del diario the Sun sobre el desastre del estadio de fútbol de Hillsborough en 1989 (en el que murieron 96 personas) provocó que muchas personas en Liverpool boicotearan el periódico, un boicot que ha durado hasta el momento" (p.6). 
municativos. Así, y debido a que los medios de comunicación y sus diferentes plataformas atraviesan gran parte de nuestra vida cotidiana, se ha prestado mucha atención a la relación entre marcos (formas de encuadre), discursos periodísticos y esfera pública (Gamson y Modigliani, 1989; Gitlin, 1980; Pan y Kosicki, 1993; Tuchman, 1980). Para esta tradición académica es fundamental ver "cómo los medios establecen el marco en el que los ciudadanos discuten los eventos públicos" (Tuchman, 1980, p. 9). En el caso específico del referéndum de la UE, se evidenció una intensa lucha discursiva en la prensa británica por construir esa "ventana" a través de la cual se interprete este fenómeno.

Varias investigaciones han explorado las posibles explicaciones detrás del Brexit. Para un importante grupo, el voto contra la UE no puede ser entendido como irracional, según se dijo abiertamente en medios de comunicación. El Brexit fue, entre otras cosas, un voto contra los llamados "expertos" y las élites ilustradas; contra la globalización, el neoliberalismo y el desarrollo desigual; contra una economía que privilegió al sector financiero y se desconectó de su estructura industrial; en resumen, fue una reacción contra el establishment y los líderes políticos que, a lo largo de varias décadas, se alejaron de las demandas de los sectores populares (Calhoun, 2017; Clarke y Newman, 2017; Evans y Menon, 2017; Glencross, 2016; Jensen y Snaith, 2016; Jessop, 2017, 2018). Según Bob Jessop (2017):

la coyuntura del Brexit reflejó una escisión de larga data en el establishment, un empeoramiento de la crisis de representación en el sistema de partidos, una crisis de autoridad en las élites políticas, una crisis de legitimidad del Estado y una crisis de hegemonía nacional-popular sobre la población. (pp. 1-2)

Además, se ha sugerido que el enfoque en el mito nacional también podría explicar, en parte, la efectividad de la campaña del Leave. ${ }^{3}$ La nostalgia "for the good old days", en lugar de la visión pragmática (costo - beneficio), promovida por los activistas del Remain, ${ }^{4}$ puede ser una de las causas detrás del resultado del referéndum (Calhoun, 2017; Glencross, 2016; Hearn, 2017; Taylor, 2017; Wenzl, 2019; Zappettini y Krzyżanowski, 2019). El nacionalismo proporcionó un recurso simbólico convincente para diseñar una narrativa que resaltara el carácter distintivo del imperio británico. En tal sentido, varias demandas sociales fueron "manchadas" por este significante nacionalista. Benedict Anderson (2006) explica: "el nacionalismo es un artefacto cultural de un tipo particular; tiene una legitimidad emocional tan profunda [que] es capaz de ser trasplantado a una gran variedad de terrenos sociales” (p. 4). En esta línea argumentativa, la UE y los inmigrantes fueron proyectados al modo del "otro extranjero", responsable del deterioro de las ya precarias condiciones de vida de los británicos (Goodwin y Milazzo, 2017). El trabajo de Khabaz (2018) analizó la intensificación de la idea de soberanía nacional a través de mantras como: "taking back control" (retomar el control). Otros marcos insertados en esta perspectiva representaron a la UE como un superestado antidemocrático y deficiente. En palabras del mismo autor: "burocracia disfuncional que no tiene una supervisión democrática adecuada” (Khabaz, 2018, p. 9).

Otros estudios se han enfocado en cómo la permanencia en la UE fue narrada al modo de una crisis por ambas facciones políticas (Bennett, 2019a; Krzyżanowski, 2019; Zappettini y Krzyżanowski, 2019). Este encuadre implicaba que se debían tomar medidas urgentes para corregir el problema: votar a favor o en contra de la membresía europea. En la misma línea, Bennett (2019b) demuestra que los valores pueden funcionar a manera de poderosos marcadores discursivos para reforzar identidades colectivas en tiempos de crisis y trastornos sociopolíticos. El autor sugirió que los discursos pro-UE tendían a mostrarse como relatos "correctos" de expertos internacionales. En este escenario discursivo, militantes euroescépticos le dieron un giro a este enfoque

Palabra en inglés que significa "salir" y que fue el nombre de la campaña que apoyaba la ruptura con la UE.

Palabra en inglés que significa "quedarse" y que fue el nombre de la campaña que apoyaba el proyecto de integración europea. 
y lo posicionaron como un ataque a un valor esencial, su libertad: "Bruselas nos dice qué hacer" (Bennett, 2019b). Charteris-Black (2019) examinó de qué forma las metáforas calentaron el tono de la discusión y enmarcaron la interpretación de los hechos a partir de sus potentes efectos discursivos. Otras investigaciones se concentraron en estudiar emociones (Breeze, 2019; Vasilopoulou y Wagner, 2017). El análisis de Vasilopoulou y Wagner (2017) examinó cómo la ira, el miedo y el entusiasmo moldearon las preferencias políticas hacia Europa.

El presente trabajo busca nutrir este debate y analiza, específicamente, cómo los periódicos británicos, con diferentes puntos de vista, encuadraron la cobertura noticiosa durante la campaña. De este modo, es posible comprender cómo un encuadre en particular podría haber influido en los votantes gracias a sus efectos discursivos. Al construir un marco interpretativo se excluyen otras formas de ver el mismo problema (Entman, 2009). Por ello, en los eventos políticos no hay significados dados de antemano, es decir, previos a la interacción discursiva. Esto dependerá de las elecciones lingüísticas adoptadas por los actores en juego, medios de comunicación en nuestro caso, y cómo estas serán decodificadas por las audiencias (Hall, 2005a). En palabras de Halliday (2017) "la construcción de la realidad es inseparable de la construcción del sistema semántico en que se halla codificada la realidad" (p.10). Es importante señalar que este trabajo no pretende medir la influencia efectiva de los mensajes de los medios en las audiencias, ni tampoco busca señalar que el resultado del referéndum responde estrictamente a los encuadres identificados. Se enfoca en analizar los marcos que se pusieron en funcionamiento durante la campaña porque son aspectos fundamentales de todo proceso político, sin embargo, forman parte de un fenómeno más complejo. Por estas razones, se propone analizar este fenómeno desde el paradigma del framing o encuadre.

\section{Aproximación metodológica}

Específicamente, se optó por los siguientes periódicos: Daily Mail, Mail on Sunday, Daily Mirror y Sunday Mirror. Estos medios fueron seleccionados porque, según estudios previos (Levy et al., 2016), representan una de las posiciones políticas en disputa durante el referéndum de la UE: proRemain (Daily Mirror y Sunday Mirror) y pro-Leave (Daily Mail y Mail on Sunday). Esto permitió comparar estrategias discursivas potencialmente diferentes y enriquecer el análisis. En cuanto a las características de los periódicos, son considerados tabloides. Según Baker et al. (2013), estos son medios "de menor tamaño (respecto a los denominados broadsheets), tienen artículos cortos, usan juegos de palabras en los titulares, tienden a centrarse más en historias nacionales, particularmente sobre celebridades, deportes y entretenimiento, y emplean un estilo de escritura informal” (p. 6). Estos diarios circulan a nivel nacional, lo que hace que sus contenidos sean potencialmente más accesibles para un universo amplio de la población; por lo tanto, podrían ser más influyentes que otros de circulación regional.

Se han incluido en el análisis sus versiones dominicales online, específicamente las publicadas los días 5, 12 y 19 de junio del 2016; es decir, las tres ediciones dominicales más cercanas al día de las elecciones. Vale aclarar que, a diferencia de otros países, los periódicos británicos tienen una edición específica que circula paralelamente los domingos, la cual se ha incluido en el estudio. Se han seleccionado los artículos que discutían explícitamente el referéndum de la UE, aquellos que en el titular o en el lead mencionaban el evento, sin importar la sección en que estaban publicados. Esto responde al interés por ver cómo los beneficios y las desventajas de la membresía de Gran Bretaña a la UE se hicieron más prominentes a través del lenguaje, independientemente del área temática.

El corpus se compone únicamente de noticias, porque se asume que el género informativo, a diferencia de otros (crónica, entrevista, opinión, etc.), se centra fundamentalmente en la transmisión de información y la descripción de hechos. Por lo tanto, en este género en particular es po- 
sible encontrar una "tensión" entre el propósito del periódico, representar un punto de vista específico del problema, y la acción de informar sobre los acontecimientos del mundo, con un cierto grado de neutralidad, para satisfacer las expectativas de sus audiencias. Finalmente, se estudiaron los titulares: 60 en total. Se ha priorizado este segmento de la noticia porque el titular es el primer elemento con el que el lector interactúa, además, es la información más destacada del artículo, donde se resume la idea central de todo el texto (van Dijk, 1988).

En cuanto al programa de análisis, se propone realizar un estudio cualitativo del corpus y se han considerado las herramientas teóricas y analíticas que ofrece el Framing Analysis (Entman, 1993; Reese, 2008; Reese et al., 2008; Van Gorp, 2010). La metodología involucra una serie de etapas que parten de un nivel macro, en el cual se identifican los principales temas, para luego examinar otros rasgos lingüísticos más específicos (dispositivos de encuadre y dispositivos de razonamiento), que contribuyen a consolidar una representación particular del referéndum. Es importante reconocer que, como en todo enfoque cualitativo, "algún nivel de subjetividad [es] inevitable" (Van Gorp, 2010, p. 90). Sin embargo, se han tomado consideraciones metodológicas para reconstruir los marcos interpretativos, o paquetes de encuadre, garantizando la confiabilidad de los hallazgos (Van Gorp, 2010). Un paquete de encuadre es "una estructura integrada de dispositivos de encuadre y una cadena lógica de dispositivos de razonamiento que demuestra cómo funciona el marco para representar un determinado tema" (Van Gorp, 2010, p. 91). Lo relativo a los dispositivos de encuadre y de razonamiento se abordará en párrafos siguientes.

En el nivel más amplio (objetivo específico 1 [OE1]), se realiza un estudio de contenido para encontrar lo que Van Gorp (2010) denomina "estructura de significado subyacente". Esto arroja una primera lectura de los datos. Específicamente, se responde a las siguientes preguntas: ¿̇uáles son los principales temas del corpus? y ¿̇cuáles son sus aspectos más repetitivos? El segundo componente (objetivo específico 2 [OE2]) involucra dos tipos de análisis, los cuales, es importante mencionar, no se ejecutan en un orden cronológico, sino que se "va y vuelve" en los datos para obtener una lista clara de todos los dispositivos utilizados. Un primer aspecto analiza cómo se contó el fenómeno político y se articuló el relato en una cadena lógica desde una visión específica del mundo. Esto se puede identificar a partir de los denominados dispositivos de razonamiento propuestos por Entman (1993). Según el autor, un encuadre define al menos dos de las cuatro funciones siguientes: 1) ¿Cuál es el problema? 2) ¿Cuáles son sus causas? 3) ¿Qué juicios morales se transmiten? y 4) ¿Cuáles son sus posibles soluciones? Este estudio se centra, específicamente, en sus dos funciones más importantes, a decir del mismo Entman (2009): definición del problema (esta condiciona el resto del encuadre), y solución propuesta (aquí se promueve el apoyo a una de las posturas en conflicto). En ciertos encuadres, y solo cuando esta función aparece en el texto y es considerada indispensable para su explicación, se incorporará un tercer criterio: causas del problema. No todos los encuadres presentan las cuatro funciones antes señaladas, la mayoría presenta dos: definición del problema y solución sugerida.

La tercera pieza del análisis presta atención a elementos más específicos en el uso del lenguaje y cómo estos dispositivos de encuadre contribuyen a diseñar un marco de interpretación particular del evento (Van Gorp, 2010). A diferencia de los dispositivos de razonamiento, que no necesariamente aparecen de manera explícita en el mensaje, sino que pueden ser intuidos a partir de la estructuración del enunciado, los dispositivos de encuadre son "aspectos manifiestos del encuadre y funcionan como indicadores demostrables del marco interpretativo" (Van Gorp, 2010, p. 91). De hecho, la forma en la que estos se articulan en el enunciado permite identificar los dispositivos de razonamiento y la cadena lógica representada.

Específicamente, el análisis se enfoca en los siguientes dispositivos, que han demostrado ser útiles para la identificación de estrategias de encuadre, debido a que contribuyen en la estructuración narrativa y retórica de la notica: metáforas conceptuales; apelaciones lógicas, emocionales 
y éticas; frases de impacto; representaciones; ejemplos históricos; elecciones léxicas y fuentes (Baden, 2018; Entman, 2009; Gamson y Lasch, 2012; Gamson y Modigliani, 1989; Pan y Kosicki, 1993; Tarín Sanz, 2017; Van Gorp, 2005, 2010; Van Gorp y Van der Goot, 2012). Es relevante notar que, si bien se analizó el corpus a partir de estos dispositivos, no todos aparecieron en las estrategias de encuadre identificadas. En concreto, este componente intenta responder a la siguiente pregunta: ¿Qué aspectos de la realidad se resaltan u oscurecen mediante el uso de dispositivos de encuadre, y cómo estos forman parte de una estrategia discursiva?

El análisis y la codificación de estas etapas sigue una lógica inductiva a partir de los procedimientos propuestos por Strauss y Corbin (2004): codificación abierta, axial y selectiva. Primero, y tomando como referencia lo propuesto por Van Gorp y Van der Goot (2012), se hizo un inventario de aquellos segmentos manifiestos del texto que estimulaban la interpretación de los eventos desde un ángulo específico, es decir, se identificaron los dispositivos de encuadre (metáforas, ejemplos históricos, elecciones léxicas, etc.). Posteriormente, se asignaron códigos cuando se consideró que un elemento de la lista seleccionada definía el problema o fomentaba una solución. Este segundo paso permitió identificar los dispositivos de razonamiento. Una vez obtenida una extensa lista de dispositivos de encuadre y razonamiento, se organizaron los códigos en categorías (codificación axial), a partir de sus similitudes y diferencias, tomando como criterio la forma en que estos contribuyen a la construcción de un argumento en torno a la permanencia del RU en la UE.

El objetivo es presentar, en una matriz organizada (Frame Matrix), una serie de paquetes de encuadre mutuamente exclusivos, que permitan evidenciar, de manera desagregada, las estrategias utilizadas por los medios para representar el evento. Finalmente, se evalúa la idoneidad de la matriz respondiendo a la siguiente pregunta: ¿̇l paquete identificado tiene una amplia lista de dispositivos de encuadre que apunten a promover un significado específico del evento? Esta pregunta se ocupa del criterio denominado por Van Gorp (2010) como "espesor de la descripción". El segundo criterio tiene que ver con la articulación lógica del relato, es decir, si la cadena de razonamiento -específicamente sus dos componentes señalados, definición del problema y posible solución-, fue suficientemente consistente entre sus partes, a partir de los segmentos del texto identificados.

\section{Análisis de contenido en Daily mail / mail on Sunday (OE1)}

En este apartado se abordan los ejes temáticos más recurrentes en el corpus y, por tal motivo, se convierten en un filtro de los datos para analizar posteriormente las estrategias de encuadre. Ello arroja una radiografía que servirá de guía para el siguiente paso del análisis. El eje más repetido gira en torno a los riesgos que implicaría dejar la UE (ver Tabla 1). Esta categoría tiene un total de 22 registros y de ella se desprenden otras subcategorías. La más recurrente (16 códigos) tiene que ver con riesgos en materia económica: apuros en el pago de cuentas hipotecarias, incertidumbre respecto al pago de pensionistas públicos, desregularización en el sistema bancario, temor a invertir en negocios inmobiliarios, posible incremento de impuestos. Vale señalar que un importante grupo de códigos dentro de esta misma subcategoría (4) habla sobre cómo el Brexit, en materia económica, tendría efectos negativos más allá de las fronteras nacionales del RU.

El segundo eje temático más frecuente tiene que ver con la inmigración. Los códigos vinculados a esta categoría giran en torno a lo siguiente: adoptar medidas para detener la inmigración; explicaciones respecto a cómo el fenómeno migratorio afecta la imagen de David Cameron y, con esto, la campaña del Remain; la migración turca y, finalmente, los discursos antinmigración elaborados por la campaña euroescéptica, especialmente por Nigel Farage, y un poster que generó gran con- 
troversia por su fuerte contenido xenofóbico. ${ }^{5}$ Otro grupo importante de códigos señala que el Brexit no tendría un efecto negativo sobre la población británica. La mayor parte de estos indica que la salida de la UE no afectaría la economía, es decir, no generaría una recesión económica, por ejemplo: las tasas de interés no se incrementarán y, a pesar de la incertidumbre, los precios de las casas ya han alcanzado un punto récord. Otros códigos dentro de esta categoría afirman que votar por el Brexit es un voto por la esperanza ("Brexit is a vote for hope") y que no hay necesidad de alarmarse en caso de una victoria del Leave ("Don't panic").

Las otras categorías tienen menos fundamentaciones. Por un lado, un grupo de códigos se enfoca en describir qué tipo de actores apoyan a cada bando político. A favor del Remain: China, jugadores de fútbol profesional, alcalde de Londres, una potente coalición de partidos políticos y la iglesia británica (5); por el Leave: Israel y John Cleese (2). ${ }^{6}$ Sin embargo, llama la atención la poca frecuencia de códigos asociados con el asesinato de la diputada laborista Jo Cox, a pesar de que su muerte se produjo el 16 de junio, tres días antes de una de las tres fechas elegidas para la recolección de datos del presente estudio.

Tabla 1. Códigos asociados. Diarios: Daily Mail / Mail on Sunday

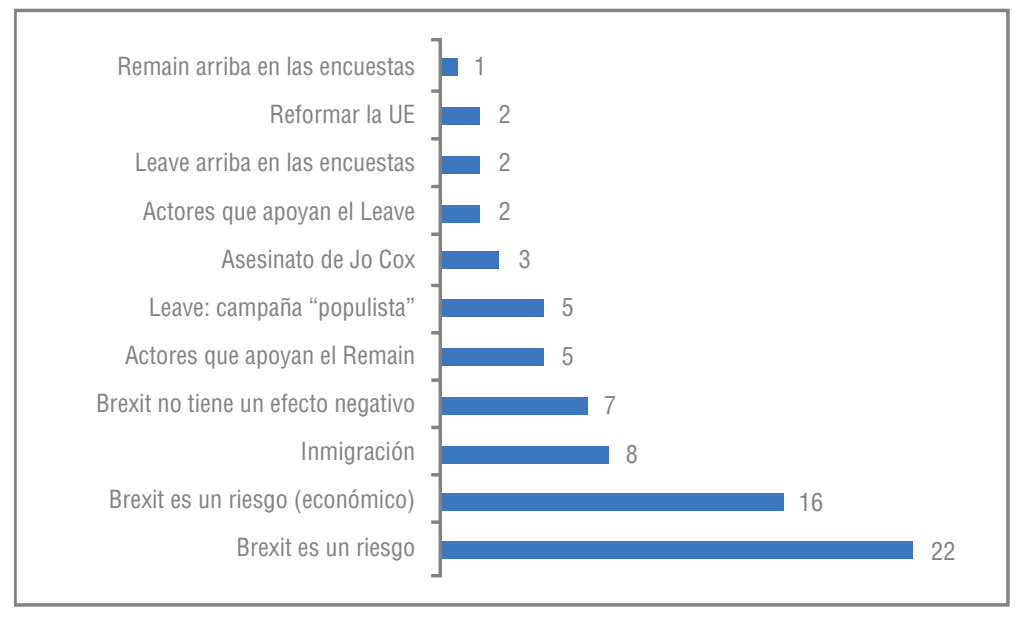

Fuente: Elaboración propia.

\section{Análisis de contenido en Daily mirror / Sunday mirror (Oع1)}

En cuanto a estos diarios, se registraron menos noticias publicadas respecto al Daily Mail y Mail on Sunday: 15 frente a 45 localizadas en el otro periódico. Al igual que en los otros dos casos analizados, representar al Brexit como una crisis es la categoría con mayor fundamentación. Se indica, por ejemplo, que dejar la UE podría subir los costos de las hipotecas; se advierte sobre los riesgos del Brexit; se anuncia que, en caso de salir, hay un 'plan de contingencia'; se señala que dejar el bloque sería devastador para la prosperidad del RU y que las siguientes generaciones tendrán que vivir con las consecuencias. La segunda categoría más reiterada habla sobre la inmigración. Sin embargo, este tema fue abordado por el diario a manera de "reacción" ante una serie de comentarios, realizados por Nigel Farage, en los que señaló que votar por el Remain podría pro-

\footnotetext{
5 En la imagen se puede ver una larga fila de inmigrantes con la siguiente consigna: "Breaking Point: the EU has failed us all" (Punto de Ruptura: la UE nos ha fallado a todos).

6 Actor y comediante británico.
} 
vocar "ataques sexuales masivos" de inmigrantes. Estos comentarios generaron mucha controversia en todo el país y se discutieron en varios medios de comunicación. Finalmente, se registraron dos códigos vinculados al asesinato de Jo Cox. Las otras categorías tienen una codificación muy baja.

Tabla 2. Códigos asociados. Diarios: Daily Mirror / Sunday Mirror

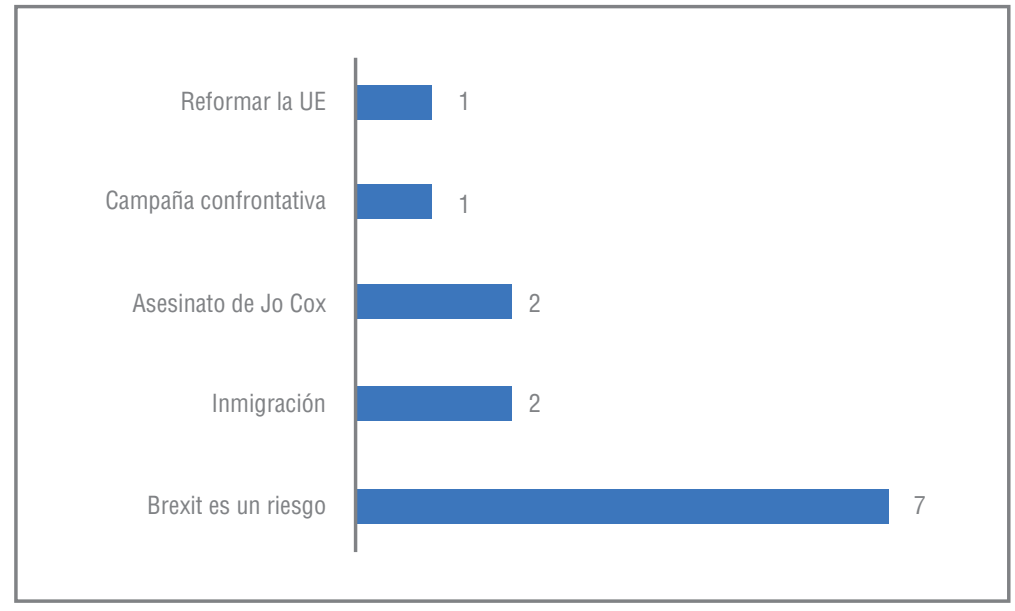

Fuente: Elaboración propia

\section{El encuadre de la crisis económica en Daily mail / mail on Sunday (Oع2)}

En cuanto al Daily Mail y Mail on Sunday, se identificaron un total de tres estrategias de encuadre. Si bien otros encuadres aparecieron en el análisis, este se ha enfocado en aquellos que cumplen con los dos criterios antes señalados (espesor de la descripción y adecuada articulación lógica) y que, por lo tanto, pueden considerarse los más prominentes. En este sentido, algunos códigos identificados en el análisis de contenido pudieron quedar fuera de la Matriz de Encuadre.

El encuadre de la crisis económica, en Daily Mail y Mail on Sunday, da relevancia a la idea de que dejar la UE afectaría la economía del RU, lo cual repercutiría sobre las condiciones de vida de la población británica. Así, se proyecta el Brexit como una "futura crisis" (Krzyżanowski, 2019). Este argumento se constituyó, en este corpus, como el principal elemento en torno al cual giró la defensa del Remain. Por un lado, esto es consistente con el análisis de contenido, pues la subcategoría que enunciaba el Brexit como un riesgo para la economía se posicionó como la más fundamentada. Por otro, esto coincide también con la literatura producida en torno al Brexit. Según varias publicaciones académicas, hubo un intento, por parte del discurso del Remain, de enfocarse en lo económico y la relación costo - beneficio (Evans y Menon, 2017; Glencross, 2016).

En cuanto a los dispositivos de encuadre pudimos observar apelaciones emocionales (pathos): "Los inversores se abstienen de los acuerdos inmobiliarios en el Reino Unido por temores al Brexit"; "Carney se prepara para emitir una nueva advertencia sobre el Brexit". 7 Mark Carney fue el director del Banco de Inglaterra durante el referéndum en el 2016. Esta institución era la encargada de dirigir la política monetaria del país. En estos ejemplos, es evidente el vínculo entre lo económico y el miedo en un mismo argumento. Se alude directamente a 'advertencias' en materia

7 Los fragmentos citados en este y los siguientes subtítulos son traducción del autor y se extraen de las noticias seleccionadas para la muestra. 
financiera y ‘temores’ para invertir. Igualmente, se identificaron apelaciones lógicas (logos): “El Brexit hará que las facturas hipotecarias se disparen en 1.50o libras esterlinas". En este caso, se intenta convencer, a través de datos y estadísticas, sobre los peligros de dejar el bloque europeo y la crisis "inevitable" atada a esta decisión.

Otro dispositivo frecuentemente utilizado fue el de la metáfora: "El golpe del Brexit en la economía podría ser peor de lo previsto"; "Después del golpe de la libra esterlina, las encuestas muestran que los británicos están muy divididos sobre la membresía en la UE”; "El Brexit [...] podría dañar la financiación del Servicio Nacional de Salud"; "El Brexit podría hacer escalar los impuestos". Las metáforas permiten representar un concepto abstracto y complejo, como la economía, a partir de algo concreto y fácil de reconocer, gracias a nuestras experiencias cotidianas: golpear, dañar o escalar. Asimismo, las elecciones léxicas también fortalecen esta estrategia: "Cameron advierte que el Brexit"; "La incertidumbre global significa más miseria para los ahorradores". En estos casos, otros términos neutros pudieron ser empleados, por ejemplo: "Cameron señala", o "La incertidumbre global generaría problemas”. Sin embargo, la elección de estas unidades léxicas (advierte y miseria) forma parte de un encuadre que opta por intensificar la idea del miedo y el imaginario de crisis. Finalmente, el escenario de crisis se extendió más allá de las fronteras británicas. Si bien estos casos no entran en la Matriz de Encuadre vale la pena mencionarlos: "El Brexit podría causar grandes problemas para bancos alemanes"; "Brexit podría aumentar la presión al alza sobre el franco suizo"; "El Brexit podría tener un efecto dominó sobre la Europa del Este".

\section{El encuadre del intruso en Daily mail / mail on Sunday (Oع2)}

Otro encuadre importante, en este caso a favor del Leave, intentó mostrar al inmigrante como un intruso y, específicamente, como un peligro para el país. En cuanto a sus dispositivos de razonamiento, se identificó el incremento de la inmigración representado como un problema para el RU, y la falta de políticas de control como el principal motivo de este fenómeno. Las metáforas utilizadas intensificaron la idea del "intruso" de una manera muy potente: "Cerrar las puertas británicas a la inmigración masiva"; "Abrir nuestras fronteras a 1,5 millones de turcos sería un riesgo". En los dos casos, se muestra al RU como un hogar, una casa o una vivienda que podría ser invadida por un intruso extranjero. Esta potente idea se nutre de un discurso patriótico que, a decir de Charteris-Black (2019), produce una especial conexión con las personas, intuitiva antes que racional, y que se construye a partir de una memoria histórica común. Por patriótico nos referimos a: "devoción a un lugar en particular y una forma de vida particular, que uno cree que es el mejor del mundo [...] El patriotismo es por naturaleza defensivo, tanto militar como cultural" (Orwell, 1945, párr. 2). Las apelaciones lógicas refuerzan la noción de gran cantidad: "1.5 millones de turcos". Finalmente, las elecciones léxicas consolidan la sensación de amenaza. El término "masivo" pudo ser eliminado del enunciado y, aun así, transmitir la misma idea: "cerrar las puertas a la inmigración". Este enfatiza el imaginario del inmigrante como un intruso y una amenaza que no se puede controlar.

\section{El encuadre del pánico exagerado, 'fear on steroids', en Daily mail / mail on Sunday (OE2)}

Este último encuadre se posiciona, al igual que el anterior, a favor de la opción del Leave. Gira en torno a la idea de la exageración por parte del Remain, al subrayar los efectos negativos, desmesurados, que podría generar la salida del bloque europeo. Esta estrategia discursiva se ubica casi como una repuesta al encuadre previo que representó al Brexit como una crisis económica. Ningún discurso se inserta en un campo de manera aislada, siempre interactúa y dialoga con otros relatos en competencia. Como bien plantea Verón (1987), "todo acto de 
enunciación política supone necesariamente que existen otros actos de enunciación, reales o posibles, opuestos al propio. En cierto modo, todo acto de enunciación política a la vez es una réplica y supone (o anticipa) una réplica” (p. 16). Varios dispositivos han permitido reconstruir este paquete de encuadre. Por una parte, las apelaciones lógicas son indicadores bastante claros: "El petróleo amplía sus ganancias, hasta un o,6 por ciento, a medida que se alivian los temores del Brexit"; "Los precios de venta alcanzaron un récord de £310K [...] a pesar del Brexit". Aquí se sustenta con argumentos lógicos (datos duros) que la economía británica, a pesar de la tensión por el referéndum, está en relativa calma. Las frases de impacto, por su parte, han sabido difundir de manera muy original y creativa esta idea: "Del proyecto miedo al proyecto pánico (From Project fear to Project panic)"; "Miedo a los esteroides (Fear on steroids)"; "Que no cunda el pánico (Don't panic)". Dentro de esta estrategia se intentó posicionar lo siguiente: si bien abandonar la UE podría traer consecuencias en materia económica, los defensores del $R e-$ main mostraron al Brexit, y sus efectos negativos, "en esteroides"; es decir, de forma exagerada. El término "esteroides", a través de una metáfora, refuerza la idea de la desproporción. Otros dispositivos enfatizaron, mediante elecciones léxicas, que el panorama no era tan dramático: "El Brexit NO provocará una recesión, pero ayudará a Gran Bretaña a prosperar"; "iQue no cunda el pánico! [...] El ministro CHRIS GRAYLING tiene este consejo reconfortante". Vale aclarar que el uso de mayúsculas fue un recurso empleado por el medio para enfatizar tal idea, al usarse en la palabra 'NO', aludiendo a la recesión económica. También en el nombre del ministro, para enfatizar la fuente. Este enunciado es definido como un consejo, catalogado, además, de reconfortante, lo que invita a tomarlo en cuenta. Se podía haber presentado como una aseveración o un simple comentario.

\section{El encuadre de la crisis económica en Daily mirror / Sunday mirror (Oع2)}

En este diario se registraron menos códigos que en el medio anterior y, por tal motivo, la reconstrucción del paquete de encuadre se enfocó en una sola estrategia que cumplía con los dos criterios antes señados: espesor de la descripción y adecuada articulación lógica. Otras estrategias de encuadre no cumplieron con una fundamentación mínima, por lo que no se tomaron en cuenta. Este encuadre repite la misma lógica que el diario anterior, es decir, intenta mostrar al Brexit como un riesgo para la prosperidad del RU. Respecto a las elecciones léxicas, varios términos intensifican la idea del miedo: "David Cameron advierte sobre los riesgos del Brexit"; "Richard Branson advierte que el Brexit podría ser devastador para la prosperidad del RU”. En estos ejemplos se pudieron utilizar otros términos más neutros, sin embargo, la elección trasmite la sensación de inseguridad, emergencia y recesión. Adicionalmente, se emplearon dos tipos de apelaciones: lógica y emocional. Entre las primeras, por ejemplo: "El Brexit podría hacer que su hipoteca cueste hasta 1.500 libras esterlinas". Aquí se sustenta con datos el escenario de crisis que se intenta construir. En cuanto a las segundas, aparecen términos sobre el Brexit como "devastador para la prosperidad del RU”; se afirma que la "gente joven [...] tendrá que vivir con las consecuencias" de este referéndum. Estos mecanismos discursivos trasladan al mundo de la intranquilidad, la ansiedad, la angustia y la inquietud.

\section{Discusión y conclusiones}

Como se ha descrito, los resultados muestran tres grandes estrategias de encuadre entre los dos diarios analizados. Este último subtítulo se centra en discutir dos de ellas: el encuadre de la crisis económica y el del intruso. Se han seleccionado estas porque el encuadre restante se inserta en la primera estrategia ya que, como se ha mencionado, se convierte en una especie de reacción frente a la idea de crisis. 
El encuadre de la crisis económica, según señala el análisis de estos datos, fue el principal argumento que intentó posicionar la campaña del Remain. Sin embargo, como explica Glencross (2016), "lo que probablemente demostró el referéndum de manera más clara fue hasta qué punto la opinión pública estaba fuera de sintonía respecto al argumento costo-beneficio del gobierno" (p. 2). Esto podría responder a algunos factores. Tomando como referencia lo propuesto por Bob Jessop $(2017,2018)$, el Brexit fue el síntoma de una crisis orgánica que arrastraba el Estado británico hacía muchos años. Había una total desconfianza en el sistema de partidos, en las autoridades políticas (nacionales y regionales) y, particularmente, en un modelo económico que había privilegiado la financiarización de la economía durante las últimas décadas. Desde mediados de los años 70, el sector industrial sufrió un fuerte descenso en sus actividades, lo que dio paso a una era postindustrial organizada en torno al sector financiero (Chang, 2010; Davis y Walsh, 2015; Taylor, 2017). Según Taylor (2017):

Entre 1979 y 1989, la inversión en servicios financieros aumentó en un 320,3\%, en comparación con un aumento del 12,8\% en la manufactura. Entre 1970 y 2010, la manufactura disminuyó del $30 \%$ del PIB al 13\% del PIB; el empleo en el sector manufacturero cayó del 35\% al 10\% del empleo total en el Reino Unido. (p. 27)

En estas circunstancias, el RU se convirtió en "la puerta financiera de Europa y en un destino offshore líder para los beneficios de las multinacionales" (Taylor, 2017, p. 27). La consolidación de la City of London como uno de los principales centros financieros de Europa y del mundo es una muestra evidente de este giro en las estructuras económicas del RU. Vale la pena mencionar que el proceso de financiarización se convirtió en una estrategia gubernamental dominante desde mediados de la década de 1970, que estuvo marcada por dos momentos clave: la intervención del Fondo Monetario Internacional (FMI) en 1976, y la consolidación del thatcherismo a partir de 1979. Estos hechos sellaron la inclusión del RU en el programa neoliberal delineado por el Consenso de Washington.

Así, este cambio en el modelo económico produjo que los intereses del capital financiero dominaran sobre los de las clases trabajadoras, lo que generó una polarización de la riqueza a favor de estos mismos centros, famosos por sus escándalos de corrupción -lavado de dinero, etc. - y sus laxas políticas de control (Jessop, 2017). El Remain, respaldado por el ex primer ministro David Cameron, encarnaba, al menos en sentido discursivo, a ese establishment antes descrito. Se había consolidado una crisis de representación y de legitimidad hacia las élites asociadas con los grupos financieros que, en el contexto del Brexit, no supieron articular un discurso coherente que coincidiera con las clases subalternas.

Otra razón que fracturó el encuadre de la crisis económica, que apostaba por el Remain, se ubica en un nivel más amplio: la crisis financiera mundial del 2008 y la crisis de la eurozona. El ciudadano británico había visto cómo, justamente estos mismos actores que ahora defendían la tesis proeuropea y hablaban de una "futura crisis económica" en el marco del Brexit -Comisión Europea, Banco Central Europeo, Fondo Monetario Internacional y jefes de Estado en Europa y el mundo, entre otros-, habían sido los protagonistas de dos momentos críticos para la realidad de la región. Estos hechos generaron una percepción negativa en la población sobre la efectividad del proyecto europeo y ayudaron a reforzar una narrativa euroescéptica (Taylor, 2017). Como afirma Liddle (2015) "si las generaciones anteriores fueron persuadidas de que Gran Bretaña no tenía otra alternativa que ‘unirse a Europa' debido al crecimiento económico que el continente suponía [...] ahora el público veía en sus pantallas de televisión un testimonio constante de una Europa fracasada y dividida" (p.10). Asimismo, la imposición de medidas de austeridad a varios países de Europa que entraron en crisis durante este período (Grecia, Portugal, España, Italia), y la ausencia de respuestas claras para atender su situación, crearon un clima de inseguridad y desconfianza en las instituciones europeas (Taylor, 2017). En tales cir- 
cunstancias, la UE fue percibida por la población como una amenaza para los derechos de las personas y las garantías sociales.

El segundo encuadre identificado, en este caso a favor de la campaña del Leave, se enfocó en la inmigración como un problema. A diferencia de la estrategia anterior, esta se orientaba de manera directa hacia una "amenaza" muy concreta: el inmigrante, representado aquí como un "intruso". La solución, por lo tanto, supondría dejar la UE para tomar medidas de manera autónoma, soberana, y evitar que ciudadanos extranjeros provenientes del centro y el este de Europa perturbaran las condiciones de vida del británico promedio. La inmigración se constituyó en un tema ampliamente debatido en el RU, sobre todo a partir del 2004, cuando 25 nuevos Estados del este y centro de Europa se adhirieron al bloque. Esto facilitó, inevitablemente, la movilidad de personas hacia el RU. Según Evans y Menon (2017), “en 2013, la ONS [Oficina de Estadísticas Nacionales, por sus siglas en inglés] estimó que la cifra real [sobre inmigración] en la región era de 50.ooo. En 2014, había casi 1,5 millones de trabajadores de Europa Central y Oriental viviendo en el Reino Unido" (p. 15). Este encuadre, como se señaló anteriormente, topa un elemento muy potente: el discurso patriótico. La idea de la nación como un hogar se convirtió en un mensaje convincente y simple, que contrastaba con los complejos argumentos en torno al impacto económico, los cuales involucraban explicaciones demasiado elaboradas por parte de "tecnócratas" y "entendidos" asociados con una élite alejada de las necesidades sociales. En esa línea, la nostalgia sobre el "imperio Británico", y ese sentimiento de "the good old days", sumado a la concepción generalizada de haber perdido el papel de potencia global tras unirse a la Comunidad Económica Europea, proporcionaron un recurso simbólico muy poderoso que permitió a los partidos políticos de extrema derecha diseñar una narrativa euroescéptica y xenófoba al destacar el carácter distintivo de la cultura británica.

A manera de conclusión, se puede señalar que hay un problema estructural, en términos económicos, que marcó los ritmos de las pugnas discursivas. Es decir, algunos encuadres no tuvieron el eco deseado debido a las brechas sociales generadas por la financiarización de la economía británica y la consolidación del proyecto neoliberal en su versión especulativa. La UE se ha convertido en un organismo que ha fortalecido estas prácticas y que, según Taylor (2017), "se debe entender como una forma de 'regionalismo abierto' a través del cual se media la relación entre los estados miembros de la UE y el globalismo neoliberal” (p. 24). Como se ha visto, se construyó un vacío entre las clases trabajadoras, insertas en el aparato industrial, y las élites económicas y culturales vinculadas a una lógica financiera, cosmopolita y global. Por tanto, catalogar a las clases subalternas como "ciegas", "ignorantes" e "incapaces" de reconocer sus "verdaderos" intereses, implica una visión simplista e inexacta del fenómeno.

En cuanto a los diarios, se observa que el Daily Mail y el Mail on Sunday presentan estrategias a favor y en contra de dejar la UE. Las posturas sobre la inmigración como un intruso y de una "crisis on steroids" son estrategias que se consolidaron en estos dos medios, y que no aparecen en el Daily Mirror y el Sunday Mirror. Mientras que la idea del Brexit como una futura crisis se repite en todos los casos.

\section{Referencias}

Anderson, B. (2006). Imagined Communities: Reflections on the Origin and Spread of Nationalism. Verso.

Baden, C. (2018). Reconstructing frames from intertextual news discourse. In P. D’ Angelo (Ed.), Doing Framing Analysis II: Empirical and teoretical Perspectives. Routledge and Taylor \& Francis Group.

Baker, P., Gabrielatos, C. \& Mcenery, T. (2013). Discourse Analysis and Media Attitudes. The Representation of Islam in the British Press. Cambridge.

Bennett, S. (2019a). 'Crisis' as a discursive strategy in Brexit referendum campaigns. Critical Discourse Studies, 16(4), 449-464. https://doi.org/10.1080/17405904.2 019.1591290 
Bennett, S. (2019b). Values as tools of legitimation in EU and UK Brexit discourses. In V. Koller, S. Kopf \& M. Miglbauer (Eds.), Discourses of Brexit (pp. 17-31). Taylor \& Francis.

Breeze, R. (2019). Emotion in politics: Affective-discursive practices in UKIP and Labour. Discourse and Society, 30(1), 24-43. https://doi.org/10.1177/0957926518801074

Calhoun, C. (2017). Populism, Nationalism and Brexit. In W. Outhwaite (Ed.), Brexit: Sociological Responses (pp. 57-76). Anthem Press.

Chang, H.-J. (2010). 23 things they don't tell you about capitalism. Penguin Group.

Charteris-Black, J. (2019). Metaphors of Brexit No: Cherries on the Cake? Palgrave Macmillan.

Clarke, J. \& Newman, J. (2017). 'People in this country have had enough of experts': Brexit and the paradoxes of populism. Critical Policy Studies, $11 \quad$ (1), 101-116. https://doi.org/10.1080/19460171.2017.1282376

Coxall, B., Robins, L. \& Leach, R. (2003). Contemporary British Politics. (4th ed.). Palgrave Macmillan. https://doi.org/10.1007/978-1-349-14821-9

D’ Angelo, P. \& Kuypers, J. A. (Eds.). (2010). Doing news framing analysis: Empirical and theoretical perspectives. Routledge and Taylor \& Francis Group. https://doi. org/10.4324/9780203864463

Davis, A. \& Walsh, C. (2015). The Role of the State in the financialisation of the UK Economy Political Studies Early View. Political Studies, n/a. https://doi.org/10. 1111\%2tf1467-9248.12198

Entman, R. M. (1993). Framing\#: Toward Clarification of a fractured paradigm. Journal of Communication, 43(4), 51-58. https://doi.org/10.1177/1369148117710799

Entman, R. M. (2009). Projections of Power: Framing News, Public Opinion, and US Foreign Policy. The University of Chicago Press. https://play.google.com/books/reader?id=SzGoHkZeoNIC\&pg=GBS.tftf4.w.3.o.o_18\&hl=es

Evans, G. \& Menon, A. (2017). Brexit and British Politics. Polity Press.

Gamson, W. \& Lasch, K. (2012). Evaluating the Welfare State. In Heckman, J. y Smith, J. (Ed.), Econometrics and Economic theory in the 20th Century (pp. 397-415). Cambridge University Press.

Gamson, W. \& Modigliani, A. (1989). Media Discourse and Public Opinion on Nuclear Power\#: A Constructionist Approach. American Journal of Sociology, 95(1), $1-\quad 37$. http://www.jstor.org/stable/2780405

Gitlin, T. (1980). The Whole World Is Whatching: mass media in the making \& unmaking of the new left. University of California Press.

Glencross, A. (2016). Why the UK Voted for Brexit. David Cameron's Great Miscalculation. In Why the UK Voted for Brexit. Palgrave Macmillan.

Goodwin, M. \& Milazzo, C. (2017). Taking back control? Investigating the role of immigration in the 2016 vote for Brexit. British Journal of Politics and International Relations, 19(3), 450-464. https:// doi.org/10.1177/1369148117710799

Hall, S. (2005a). Encoding/Decoding. In S. Hall, D. Hobson, A. Lowe, \& P. Willis (Eds.), Culture, Media, Language (pp. 117-127). Routledge.

Hall, S. (2005b). Introduction to Media Studies at the Centre. In S. Hall, D. Hobson, A. Lowe, \& P. Willis (Eds.), Culture, Media, Language (pp. 104-116). Routledge.

Halliday, M. A. K. (2017). El lenguaje como semiótica social. La interpretación social del lenguaje y del significado. Fondo de Cultura Económica.

Hearn, J. (2017). Vox populi: Nationalism, Glogalization and the Balance of Power in the Making of Brexit. In W. Outhwaite (Ed.), Brexit: Sociological Responses (pp. 19-30). Anthem Press.

Hinde, S. (2017). Brexit and the Media. Hermès, La Revue, 77(1), 80-86. https://doi .org/10.1111/1467923X.12296

Jackson, D., Thorsen, E. \& Dominic, W. (Eds.). (2016). EU Referendum Analysis 2016: Media, Voters and the Campaign. Early reflections from leading UK academics. The Centre for the Study of Journalism, Culture and Community. Bournemouth University.

Jensen, M. D. \& Snaith, H. (2016). When politics prevails: the political economy of a Brexit. Journal of European Public Policy, 23(9), 1302-1310. https://doi.org/10. 1080/13501763.2016.1174531

Jessop, B. (2017).The Organic Crisis of the British State: Putting Brexit in its place. Globalizations, 14(1), 133-141. https://doi.org/10.1080/14747731.2016.1228783 
Jessop, B. (2018). Neoliberalization, uneven development, and Brexit: further reflections on the organic crisis of the British state and society. European Planning Studies, 26(9), 1728-1746. https://doi.org/10.1080/09654313.2018.1501469

Khabaz, D. (2018). Framing Brexit: the role, and the impact, of the national newspapers on the EU referendum. Newspaper Research Journal, 39(4), 496-508. https://do i.org/10.1177/0739532918806871

Kovecses, Z. (2010). Metaphor: A Practical Introduction. Oxford University Press. https://doi.org/10.1023/A:1023919116538

Krzyżanowski, M. (2019). Brexit and the imaginary of 'crisis': a discourse-conceptual analysis of European news media. Critical Discourse Studies, 16(4), 465-490. https://doi.org/10.1080/17405904.2019.1592001

Lakoff, G. M. J. (2003). Metaphors we live by. The University of Chicago Press.

Levy, D. A. L., Aslan, B. \& Bironzo, D. (2016). Uk Press Coverage of the Eu Referendum. Reuters Institute for the Study of Journalism. https:// reutersinstitute.politics.ox.ac.uk/our-research/uk-press-coverage-eu-referendum

Liddle, R. (2015). The Risk of Brexit\#: The Politics of a Referendum. Rowman \& Littlefield International.

Maccaferri, M. (2019). Splendid isolation again? Brexit and the role of the press and online media in renarrating the European discourse. Critical Discourse Studies, 16(4), 389-402. https://doi.org/10.1080/17405904.2019.1592766

Marsh, D. (2018). Brexit and the politics of truth. British Politics, 13(1), 79-89. https://doi.org/10.1057/s41293-018-0076-x

Orwell, G. (1945). Notes on Nationalism. https://www.resort.com/ prime8/Orwell/ nationalism.htmlt

Pan, Z. \& Kosicki, G. M. (1993). Framing analysis: An approach to news discourse. Political Communication, 10, 55-75. https://doi.org/10.1080/10584609.1993.9962963

Payne, R. (2018). Brexit and the British Media. The Round Table, 107(1), 109-110. https://doi.org/10.1080/00358533.2018.1424083

Reese, S. D. (2008). Prologue. Framing Public Life: A Bridging Model for Media Research. In S. D. Reese, O. H. Gandy Jr. \& A. E. Grant (Eds.), Framing Public Life: Perspectives on Media and Our Understanding of the Social World. Lawrence Erlbaum Associates. https://doi.org/10.4102/sajcd.v53i1.198

Reese, S. D., Gandy Jr, O. H. \& Grant, A. E. (Eds.). (2008). Framing Public Life: Perspectives on Media and Our Understanding of the Social World. Lawrence Erlbaum Associates.

Seaton, J. (2016). Brexit and the Media. The Political Quarterly, 87(3), 333-337. https://doi.org/10.1111/1467-923X.12296

Strauss, A. \& Corbin, J. (2004). Bases de la investigación cualitativa. Técnicas y procedimientos para desarrollar la teoría fundamentada. Editorial Universidad de Antioquia.

Tarín Sanz, A. (2017). The Ethnic Russian as an Enemy of Islam: Frame Analysis of the Kavkaz Center News Agency (2001-2004). Civil Wars, 19(4), 495-516. https://doi.org/10.1080/13698249.2017.1396112

Taylor, G. (2017). Understanding Brexit: Why Britain voted to leave the European Union. Emerald publishing.

Tuchman, G. (1980). Making News: A Study in the Construction of Reality. The Free Press.

van Dijk, T. A. (1988). News As Discourse. Lawrence Erlbaum Associates. https://doi. org/10.4324/9780203062784

Van Gorp, B. (2005). Where is the frame?: Victims and intruders in the belgian press coverage of the asylum issue. European Journal of Communication, 20(4), 484-507. https://doi.org/10.1177/0267323105058253

Van Gorp, B. (2010). Strategies to Take Subjectivity Out of Framing Analysis. In P. D’ Angelo \& J. A. Kuypers (Eds.), Doing news framing analysis: Empirical and theoretical perspectives (pp. 84-109). Routledge and Taylor \& Francis Group.

Van Gorp, B. \& Van der Goot, M. J. (2012). Sustainable Food and Agriculture: Stakeholder's frames. Communication, Culture \& Critique, 5(2), 127-148. https://doi.org/10.1111/j.1753-9137.2012.01135.x 
Vasilopoulou, S. \& Wagner, M. (2017). Fear, anger and enthusiasm about the European Union: Effects of emotional reactions on public preferences towards European integration. European Union Politics, 18(3), 382-405. https://doi.org/10.1177/ 1465116517698048

Verón, E. (1987). La Palabra Adversativa: Observaciones sobre la enunciación política. En E. Verón, L. Arfuch, M. M. Chirico, E. De Ipola, N. Goldam, M. I. González \& Ó. Landi (Eds.), El discurso político: Lenguajes y acontecimientos (pp. 11-26). Hachette.

Wenzl, N. (2019). 'This is about the kind of Britain we are': National identities as constructed in parliamentary debates about EU membership. In V. Koller, S. Kopf \& M. Miglbauer (Eds.), Discourses of Brexit (pp. 32-47). Routledge and Taylor \& Francis Group.

Zappettini, F. \& Krzyżanowski, M. (2019). The critical juncture of Brexit in media \& political discourses: from national-populist imaginary to cross-national social and political crisis. Critical Discourse Studies, 16(4), 381-388. https://doi.org/10.10 80/17405904.2019.1592767 